\title{
Transconductance-Mode Gain Controllable First Order Allpass Filter
}

\author{
Piya Supavarasuwat ${ }^{\mathrm{a}, *}$, Montree Kumngern ${ }^{\mathrm{a}}$, Winai Jaikla ${ }^{\mathrm{b}}$ \\ ${ }^{a}$ Department of Telecommunications Engineering Faculty of Engineering, \\ King Mongkut's Institute of Technology Ladkrabang Bangkok 10520, Thailand \\ ${ }^{\mathrm{b}}$ Department of Engineering Education Faculty of Industrial Education, \\ King Mongkut's Institute of Technology Ladkrabang Bangkok 10520, Thailand
}

*Corresponding Author: piya.su@kmitl.ac.th

\begin{abstract}
New first order allpass filter (APF) in transconductance mode, constructed from 1 DDCCTA, 1 resistor and grounded capacitor, is presented. The transconductance gain and phase shift can be independently controlled. High input and high output impedances are achieved which make the circuit to be easily cascaded without additional buffers. The magnitude of output current can be electronically adjusted. The operation of the proposed filter has been verified through simulation results using 0.5um MIETEC CMOS technology which confirm the theoretical analysis.
\end{abstract}

Keywords: Transconductance-mode, Allpass filter, Differential difference current conveyor transconductance amplifier (DDCCTA).

\section{Introduction}

"A first order all-pass filter or phase shifter is a very useful function blocks of many analog signal processing applications. It is frequently used in many active circuits such as, phase shifters, oscillators and high-Q band-pass filters ${ }^{(1)}$. The first order all-pass filter with gain controllability is very useful for design in many analog circuits to avoid the use of external amplifiers, for examples quadarture oscillator ${ }^{(2)}$ and multiphase sinusoidal oscillator ${ }^{(3)}$ with non-interactive control for oscillation condition and oscillation frequency." (4)

"The synthesis and design of analog filters using modern electronically controllable active building blocks (ABBs) provide flexibility and convenience for designer. These filters can be easily controlled by microcomputer or microcontroller. Also some filter circuits, employing active building blocks, can avoid the use of external resistors." (5) The differential difference current conveyer transconductance amplifier (DDCCTA) was introduced ${ }^{(6)}$. "This active building block (ABB) with three voltage inputs and two kinds of output current is constructed from the well-known advantages of the differential difference current conveyer (DDCC) and the operational transconductance amplifier (OTA). It provides the facility for the implementation of voltage and current mode signal processing. Moreover, it transconductance gain (gm) can be electronically tuned which is easy to use in the modern microcontroller or microprocessor based electronic systems." (7)

In this paper, the transconductance mode first order allpass filter using DDCCTA as active element is presented. The proposed filter offers the following advantageous features. The proposed APF also exhibits high output input impedances, which is easy cascading in the transconductance-mode operation. The performances of the proposed circuit are illustrated by PSpice simulations, they show good agreement with the calculation.

\section{Circuit Description}

\subsection{Differential difference current conveyor transconductance amplifier}

Fig. 1. shows the circuit symbol of the DDCCTA. The internal construction of CMOS DDCCTA is illustrated in Fig. 2. The terminal relations of an ideal DDCCTA can be described by the following expressions: 


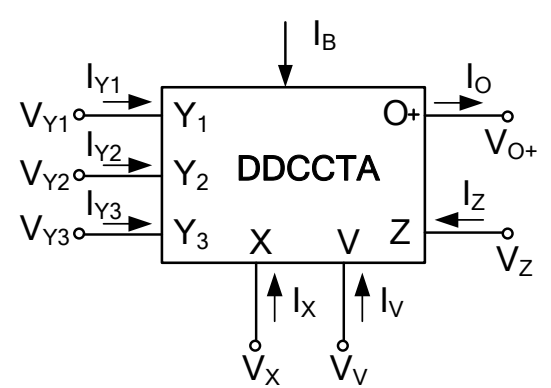

Fig. 1. Circuit symbol of the DDCCTA.

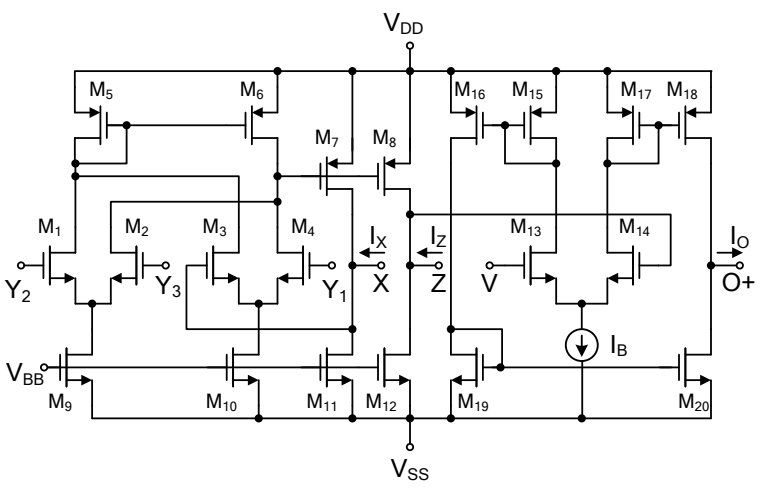

Fig. 2. Internal construction.

$$
\begin{aligned}
& I_{y 1}=I_{y 2}=I_{y 3}=0 ; \\
& V_{x}=V_{y 1}-V_{y 2}+V_{y 3} ; \\
& I_{z}=I_{x} ; \\
& I_{o}=g_{m}\left(V_{Z}-V_{V}\right) .
\end{aligned}
$$

For CMOS DDCCTA, the gm is given as

$$
g_{m}=\sqrt{\mu C_{o x} \frac{W}{L} I_{B}} .
$$

where $\mu$ is the mobility of the carrier for NMOS transistors (M13 and M14 in Fig. 2), Cox is the gate oxide capacitance per unit area, $\mathrm{W}$ and $\mathrm{L}$ are the channel width and channel length, respectively.

\subsection{Proposed filter configuration}

The proposed transconductance mode first order allpass filter is shown in Fig. 3.

It consists of one DDCCTA, single resistor and single grounded capacitor which is attractive to fabricate in monolithic chip. The proposed filter has voltage as input and current as output. The high input and output impedances are achieved. So, it can directly drive external load or connect to other circuit without any external buffer devices. The admittance transfer function is as follows:.

$$
\frac{I_{\text {out }}}{V_{\text {in }}}=g_{m}\left(\frac{S C R-1}{S C R+1}\right)
$$

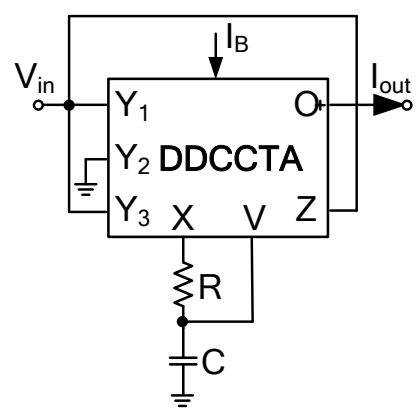

Fig. 3. Proposed filter configuration.

From Eq3, the phase response, gain response and natural frequency are written as

$$
\begin{gathered}
\phi=\pi-2 \tan ^{-1}(\omega C R), \\
G(j \omega)=g_{m} \\
\omega_{0}=\frac{1}{C R} .
\end{gathered}
$$

It is found from Eq. 4 - Eq. 6 that the gain and phase responses can be independently controlled. Also, the gain response can be electronically tuned.

\subsection{Non-idea case}

Taking into consideration the DDCCTA non-idealities, the relationship of the terminals given in Eq. 1 can be rewritten as.

$$
\begin{aligned}
& I_{y 1}=I_{y 2}=I_{y 3}=0 ; \\
& V_{x}=\beta_{1} V_{y 1}-\beta_{2} V_{y 2}+\beta_{3} V_{y 3} ; \\
& I_{z}=\alpha I_{x} ; \\
& I_{o}=g_{m}\left(V_{Z}-V_{V}\right)
\end{aligned}
$$

where $\beta 1, \beta 2$ and $\beta 3$ are, respectively, the non-ideal voltage transfer gains between $\mathrm{Y} 1-\mathrm{X}, \mathrm{Y} 2-\mathrm{X}$ and $\mathrm{Y} 3-\mathrm{X}$ terminals and $\alpha$ is the non-ideal current transfer gain between X-Z terminals of the DDCCTA. These non-ideal gains slightly differ from unity by voltage-tracking error and current-tracking errors of the DDCCTA. Thus, the admittance transfer function of the proposed filter in Fig. 3 is rewritten as:

$$
\frac{I_{\text {out }}}{V_{\text {in }}}=g_{m}\left(\frac{s C R+1-\beta_{1}-\beta_{3}}{s C R+1}\right) .
$$

The phase response, gain response and natural frequency are written as 


$$
\begin{gathered}
\phi=\pi-\tan ^{-1}\left(\frac{\omega C R}{\beta_{1}+\beta_{3}-1}\right)-\tan ^{-1}(\omega C R), \\
G(j \omega)=g_{m} \sqrt{\frac{(\omega C R)^{2}+\left(1-\beta_{1}-\beta_{3}\right)^{2}}{(\omega C R)^{2}+1}} \\
\omega_{0}=\frac{1}{C R} .
\end{gathered}
$$

\section{Simulation Results}

To verify the theoretical analysis, the proposed transconductance mode first order allpass filter in Fig. 3, was simulated with PSPICE simulation using CMOS schematic of DDCCTA as given in Fig. 2. The PMOS and NMOS transistors have been simulated by respectively using the parameter of a $0.5 \mu \mathrm{m}$ MIETEC CMOS technology ${ }^{(8)}$. The optimal aspect ratios of PMOS and NMOS transistor are listed in Table I. The circuit was biased with $\pm 2 \mathrm{~V}$ and $\mathrm{V}_{\mathrm{BB}}=-1 \mathrm{~V}$. The active and passive components are chosen as: $\mathrm{I}_{\mathrm{B}}=100 \mu \mathrm{A}, \mathrm{R}=10 \mathrm{k} \Omega$ and $\mathrm{C}=$ $10 \mathrm{pF}$. With this condition, the power consumption is about $3.71 \mathrm{~mW}$. The simulated natural frequency is about 1.57 MHz. The simulated gain and phase responses of the filter are given in Fig. 4.

Table 1. Transistor aspect ration of DDCCTA.

\begin{tabular}{|c|c|c|}
\hline MOS Transistors & $\mathbf{W}(\boldsymbol{\mu m})$ & $\mathbf{L}(\boldsymbol{\mu m})$ \\
\hline $\mathrm{M}_{1}-\mathrm{M}_{4}$ & 1.8 & 0.7 \\
\hline $\mathrm{M}_{5}-\mathrm{M}_{6}$ & 20 & 0.7 \\
\hline $\mathrm{M}_{7}-\mathrm{M}_{8}$ & 20 & 0.7 \\
\hline $\mathrm{M}_{9}-\mathrm{M}_{10}$ & 5.2 & 0.7 \\
\hline $\mathrm{M}_{11}-\mathrm{M}_{12}$ & 58 & 1.0 \\
\hline $\mathrm{M}_{13}-\mathrm{M}_{14}$ & 58 & 0.7 \\
\hline $\mathrm{M}_{15}-\mathrm{M}_{20}$ & 5 & 0.7 \\
\hline
\end{tabular}

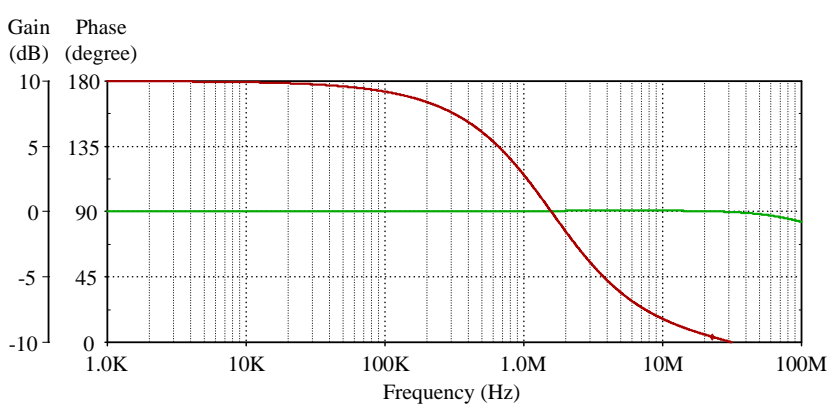

Fig. 4. Gain and phase response.

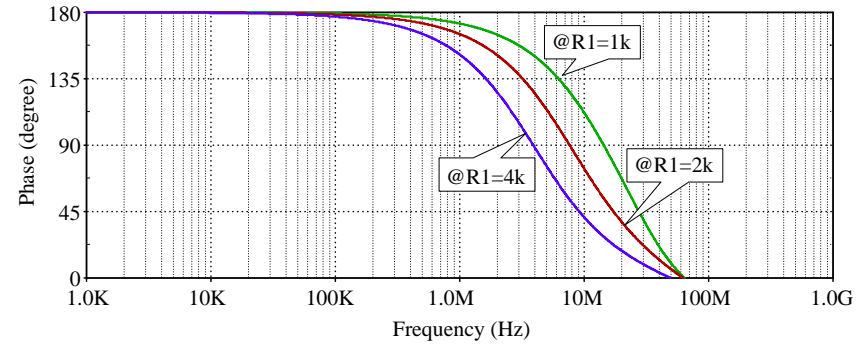

Fig. 5. phase response for different value of $\mathrm{R}$.

It can be found that the simulated gain and phase responses are slightly deviated from ideal responses due to the error terms as expressed in Eq. 9. Phase response for different value of $R$ is shown in Fig. 5. This result confirms that the phase response can be tuned by $\mathrm{R}$ as shown in Eq. 4.

The time-domain response of the proposed filter is shown in Fig. 6 where a sine wave voltage of $500 \mathrm{mVp}-\mathrm{p}$ amplitude and $1.5 \mathrm{MHz}$ is applied as the input to the filter and the output currents for different values of $\mathrm{I}_{\mathrm{B}}$ are shown. It is seen that the output current can be electronically/ independently adjusted from its phase shift as expressed in Eq. 5. The output current in time domain for different value of $\mathrm{R}$ is shown in Fig. 7. In this result, the value of $\mathrm{R}$ is changed to $1 \mathrm{k} \Omega, 3 \mathrm{k} \Omega$ and $5 \mathrm{k} \Omega$ and the phase of the output current is respectively shifted to $168.1^{\circ}, 146.4^{\circ}$ and $126.9^{\circ}$. It is found that the phase response can be tuned by $\mathrm{R}$ as shown in Eq. 4. The dependence of the output harmonic distortion of output current on input voltage amplitude is shown in Fig. 8. It can obtain from Fig. 8. that the THD is about $5.79 \%$ when the input signal is increased to $500 \mathrm{mV}$ (peak).

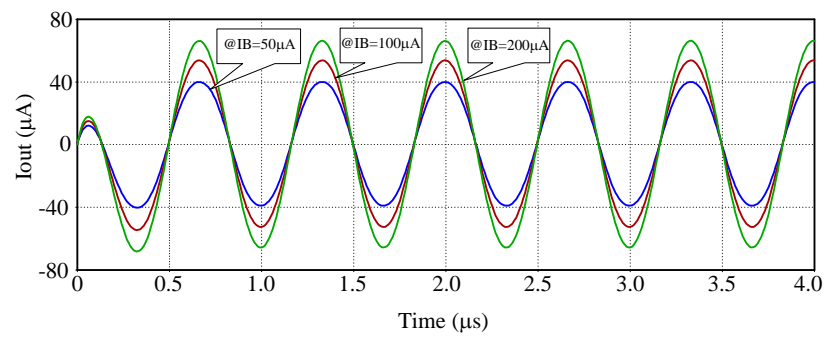

Fig. 6. Output current for different value of $\mathrm{I}_{\mathrm{B}}$.

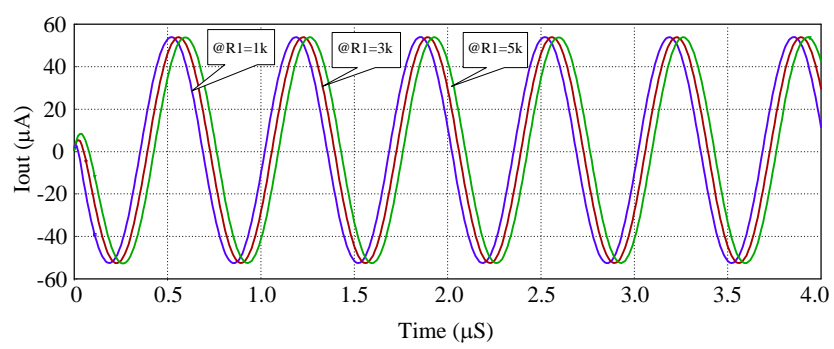

Fig. 7. output current for different value of R. 


\section{Conclusions}

An electronically tunable transconductane-mode first-order allpass filter with gain controllability has been introduced via this paper. It consists of 1 DDCCTA, 1 resistor and 1 grounded capacitor. So it is easy to fabricate in IC form to use in battery-powered or portable electronic equipments such as wireless communication devices. The PSpice simulation results were depicted, and agree well with the theoretical anticipation.

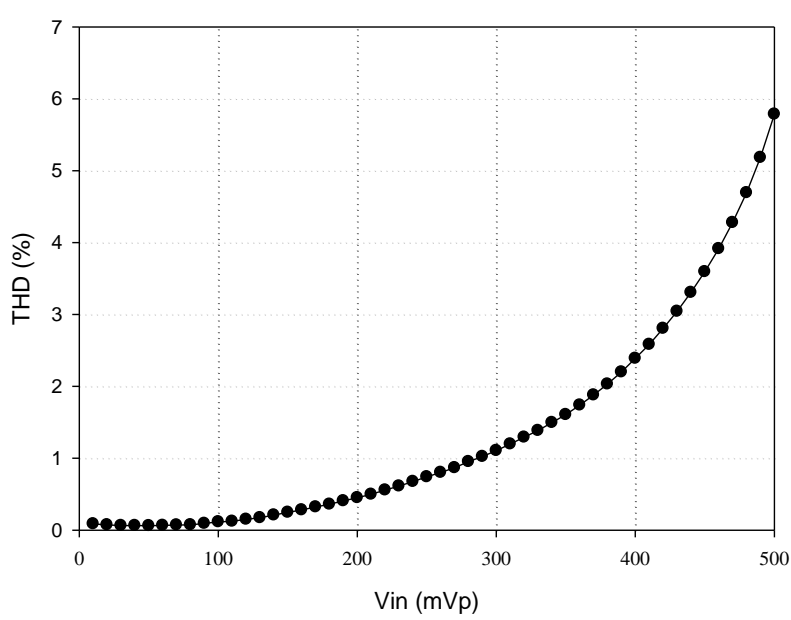

Fig. 8. Dependence of the output harmonic distortion of output current on input voltage amplitude

\section{References}

(1) R. Schauman, and E. Valkenburg. "Design of analog filters”. Oxford University Press. New York, 2001

(2) A.U. Keskin, and D. Biolek. "Current mode quadrature oscillator using current differencing transconductance amplifiers (CDTA)". IEE Proc.-Circuits Devices Syst., vol. 153, No. 3, pp. 214-218, 2006

(3) W. Jaikla, and P. Prommee. "Electronically tunable current-mode multiphase sinusoidal oscillator employing CCCDTA-based allpass filters with only grounded passive elements", Radioengineering, , vol. 20, No. 3, pp. 594-599, 2011

(4) W. Jaikla, A. Noppakarn, and S. Lawanwisut. "New Gain Controllable Resistor-less Current-mode First Order Allpass Filter and its Application", Radioengineering, vol. 21, No. 1, pp. 312-316, 2012

(5) W. Ninsraku, D. Biolek, W. Jaikla, S. Sriripongdee, and P. Suwanjan. "Electronically controlled high input and low output impedance voltage mode multifunction filter with grounded capacitors", AEU - International
Journal of Electronics and Communications, vol. 68, No. 2, pp. 1145-1264, 2014

(6) N. Pandey and S.K. Paul. "Differential difference current conveyor tranconductance amplifier : A new analog building block for signal processing", Journal of Electrical and Computer Engineering, pp. 1-10, 2011

(7) P. Maneedang, W. Jaikla, and P. Suwanjan. "High input impedance three-inputs single-output voltage-mode multifunction biquadratic filter with independent tuning using DDCCTAs", 6th International Science, Social Sciences, Engineering and Energy Conference, 2014.

(8) O. Channumsin and W. Tangsrirat. "Single-input four-output voltage-mode universal filter using single DDCCTA”, Microelectronics Journal, vol. 44, No. 12, pp. 1084-1091, 2013 